\title{
Decision procedure of some relevant logics: a constructive perspective
}

\author{
Jacques Riche \\ Department of Computer Sciences, \\ Katholieke Universiteit Leuven, \\ 200A Celestijnenlaan, B-3001 (Belgium) \\ riche@cs.kuleuven.ac.be
}

ABSTRACT. Some investigations into the algebraic constructive aspects of a decision procedure for various fragments of Relevant Logics are presented. Decidability of these fragments relies on S. Kripke's gentzenizations and on his combinatorial lemma known as Kripke's lemma that B. Meyer has shown equivalent to Dickson's lemma in number theory and to his own infinite divisor lemma, henceforth, Meyer's lemma or IDP.

These investigations of the constructive aspects of the Kripke's-Meyer's decision procedure originate in the development of Paul Thistlewaite's "Kripke" theorem prover that had been devised to tackle the decision problem of the Relevant Logic $\mathbf{R}$.

A. Urquhart's pen and paper solution that relies on a sophisticated algebraic and geometric treatment of the problem shows the usefulness of an algebraic approach in Logic. Here, the study of the constructive aspects of the Kripke-Meyer decision procedure relies on various algebraic constructive results in the theory of polynomials rings.

KEYWORDS: constructivity, decision procedure, dickson's, kripke's, meyer's lemmas, hilbert basis, polynomial rings, ideal membership problem, word problem.

\section{Introduction: another ancestor of B. Meyer's IDP}

In 1887, J. J. Sylvester was in Toulouse to talk about Hamilton's numbers. He had also the opportunity to discuss his current research in pure number theory, on primality, divisibility, factorization in primes etc. Various papers published in the "Comptes Rendus", 1887-88, report his results on perfect numbers. One of them starts with a question already raised by Euler, "Are there any perfect odd numbers?". In another article, in connection with the theory of perfect numbers, he proposed to call 'Fermatian' some expression $\theta^{i}-1$, whose properties he deduced from Fermat's theorem (if $\left.p \nmid a, a^{p-1} \equiv 1(\bmod p)\right)$, “en l'honneur du grand et surprenant Fermat, dont j'ai vu 
avec une émotion indicible gravés sur le buste au musée de Toulouse les mots qui lui étaient adressés par Blaise Pascal: 'Au plus grand homme de l'Europe'...".

It is in a paper on the finiteness of odd perfect numbers with distinct prime factors, where he proves anew some of Sylvester's results, that Dickson proposed his now famous lemma and showed it to be a consequence of Hilbert's finite basis theorem.

For this reason, in [RIC 98], we wrote that Gordan should be considered as the father of Dickson's lemma. As far as content is concerned. But with respect to the form, J. J. Sylvester was the first to make a number-theoretic suggestion for the solution of the invariant theory problem.

Invariant theory, the search for invariant algebraic expressions or forms, that Cayley called 'quantics', was a major domain of research in mathematics during the second half of $19^{\text {th }}$ century. The general form of a binary (i.e. with 2 variables) quantic of order $n$ is $a_{0} x^{n}+a_{1} x^{n-1} y+a_{2} x^{n-2} y^{2}+\ldots+a_{n} y^{n}$. The invariants are the forms that are left unchanged under linear transformation of the $x, y$ variables. When the variables $x, y$ appear in the invariants, the forms are called covariants.

Gordan (1868) proved constructively that the invariants and covariants of binary forms can be expressed in terms of a finite number of irreducible invariants and covariants. In other words, his theorem says that the number of irreducible solutions in positive integers of a system of homogeneous linear equations is finite. This finite number will later be called the Hilbert's basis, following Hilbert's general proof of the theorem ${ }^{1}$ which holds for forms in any number of variables. This proof is a mere existence proof and is considered to mark a fundamental change of attitude in algebra with the recognition and acceptance of abstract non-effective methods. Nevertheless, it is not quite correct to claim that, although it opened a new era in mathematics, calculation was no longer required.

Indeed, in the $37^{\text {th }}$ lecture of his Introductory Course on Invariant Theory, (1897), Hilbert said that "With each mathematical theorem, three things are to be distinguished. First, one needs to settle the basic question of whether the theorem is valid; one has to prove its existence, so to speak. Second,... how many operations to carry out the assertion of the theorem. Kronecker emphazised the question of a finite number of steps. Third, it has to be actually carried out...". That means that, even though there was a change of attitude and an adoption of abstract methods (which will only be completed by E. Noether's work in the 1920's), it was not in opposition to constructive and computational ones. Hilbert even recognized that, in the case of his theorem, compliance wih the second and third requirements was a problem although, in 1893, he had given a finite algorithm computing the invariants for all classical groups [HIL 93].

1. Let $\mathcal{F}_{1}, \mathcal{F}_{2}, \mathcal{F}_{3}, \ldots$ be an infinite sequence of forms in the $n$ variables $x_{1}, x_{2}, x_{3}, \ldots$ Then there always exists an integer $m$ such that each form in the sequence can be expressed as $\mathcal{F}=$ $\mathcal{A}_{1} \mathcal{F}_{1}+\mathcal{A}_{2} \mathcal{F}_{2}+\cdots+\mathcal{A}_{m} \mathcal{F}_{m}$, where $\mathcal{A}_{1}, \mathcal{A}_{2}, \ldots, \mathcal{A}_{m}$ are suitable forms of the same $n$ variables [HIL 90]. 
Returning to Sylvester, the relation between invariants and odd perfect numbers is now clear. It comes from the consideration of binary forms of odd degree. Having deduced the complete systems of groundforms for the cubic and quartic binary forms and proved their completeness by a method, first discovered by Cayley, that would lead to what "he had in view when he originated it, namely, a proof of Gordan's theorem by ordinary algebra", Sylvester [SYL 82] adds that he sees "looming in the not too far distance such a proof, depending ultimately upon the fact that a certain succession of increasing multiplets, subject to stated laws of limitation, not being capable of being indefinitely produced". Later on, he expressed that fact as

THEOREM 1. - A succession of integers or n-vectors of integers of which no one is a multiple of one nor the sum of the multiples of two others cannot be continued ad infinitum.

If the number of groundforms of quantics were infinite, then there would exist some infinite succession of products, which is impossible by the theorem.

In this paper, we will be concerned with two successors of Sylvester's theorem, Kripke's [AND 75] and Meyer's lemmas that play an essential role in the decision procedures of decidable fragments of the major Relevant Logics (particularly, $\mathbf{R}_{\rightarrow}$ ). Various relationships of Kripke's lemma with other similar results, among them, Hilbert's finite basis, Higman's lemma, Kruskal's theorem and Meyer's Infinite (Relevant) Division Principle $(I D P)$ and the combinatorics underlying the decision procedures have been examined elsewhere in [MEY 01], [RIC 98].

We recall Meyer's definition of relevant divisibility defined on the positive integers $\mathbb{N}$ and his IDP lemma stated for the commutative monoid $\mathbb{N}_{k}$ finitely generated by the first $k$ primes:

DEFINITION 2 (RELEVANT DivisiBILITY). - Let primeset be a function whose application to any positive integer $n$ yields the set of the prime divisors of $n$. Let $\left.\right|_{r}$ and $\mid$ be the relevant and ordinary divisibility relations. Then, $\left.m\right|_{r} n$ iff :

(i) $m \mid n$ and

(ii) $\operatorname{primeset}(m)=\operatorname{primeset}(n)$.

That is, all same factors are used with their exponents at least as great in $n$ as in $m$.

Lemma 3 (INFINITE Division PRINCIPLE). - Let $A_{n}$ be any infinite subset of $\mathbb{N}_{k}$. Then there is an infinite subset $A_{n}^{\prime}$ of $A_{n}$ and a member, a, of $A_{n}^{\prime}$ s.t. for all $b \in A_{n}^{\prime},\left.a\right|_{r} b$.

In the following, some investigations into the constructive aspects of a decision procedure based on these lemmas are reviewed. This research was performed in the context of automated theorem proving for Relevant Logics [RIC 91a] in order to gain more insight into the decidable logic $\mathbf{L R}$ and its decision procedure implemented by P. Thistlewaite in his theorem prover "Kripke" [THI 88]. This theorem prover had been developed as part of the attempts to solve the decision problem of the logic $\mathbf{R}$. 
A better understanding of the logical and algebraic engineering could allow to enhance the theorem prover and eventually reach the old goal of finding the elusive associative connective for $\mathbf{R}$-to which we will come in the next section- as well as to tackle other problems in these logics, for example, the decision problem of $\mathbf{T}_{\rightarrow}$ based on IDP in an appropriate almost commutative algebraic structure.

These investigations also throw light on the algebra underlying various procedures used in the undecidability and complexity proofs of A. Urquhart as well as on the finitization lemmas mentioned above.

\section{Beautiful problems of relevant logic}

Answering a question of Natasha Kurtonina at the first WCP about 'beautiful problems' still waiting for a solution in Relevant Logics, R. K. Meyer" asked for "a clean and convincing proof of the undecidability of $\mathbf{R}$ and/or other Relevant Logics" because A. Urquhart's proof of undecidability in [URQ 84], [AND 92] lacks "the immediacy that we seek, and usually find, in arguments to unsolvability".

One hint that might work, R. Meyer writes, would be to find a free semigroup operation definable in $\mathbf{R}$. Refering to the unsuccessful attempts to find one, he concludes “...we applied a 'needle in the haystack' method, which failed..."'. Indeed, in order to verify the conjectured undecidability of the logic $\mathbf{R}$, the undecidable word problem for semigroups was coded into $\mathbf{R}$ through a translation of the usual semigroup operations into the $\mathbf{R}$ connectives. The problem was to find a connective corresponding to the semigroup multiplication operator which is associative, but not commutative nor idempotent, that is, to find a free associative connective in $\mathbf{R}$. If 32 formulae that define the possible candidates for this connective were proved in $\mathbf{L R}$, that would mean that the corresponding connective is not the free connective wanted for R. A. Urquhart's proof of the undecidability of $\mathbf{R}$ interrupted this research. But the problem of defining an associative connective has remained a challenge.

Although he does not find his proof in need of being cleaned up, A. Urquhart [ibidem] admits that "a more direct solution would be nice". Related to this issue, he poses two problems: whether there is a well-defined freely associative connective in $\mathbf{R}$ and whether the two variables fragment of $\mathbf{R}$ is decidable. He conjectures that both questions have negative answers.

With respect to the first problem, running "Kripke" on a parallel supercomputer, the $\mathrm{CM} 2$, using $232 \mathrm{k}$ virtual processors and large databases of results, 24 of the 32 formulae mentioned above had relatively short and verifiable proofs. In order to prove the remaining formulae, various experiments using various selection functions in the execution of the proof theory as well as other empirical tricks were tried but without

2. Communicated in the relevant-logic newsgroup, October 161997.

3. More on the method can be found in [THI 88] where P. Thistlewaite, R. Meyer and

M. McRobbie develop their approach based on the first author's thesis. 
any means of verifying the correctness of the (positive) results. Finding short and surveyable proofs is still an open problem.

Essentially, it appeared that a quick proof hangs on an appropriate selection of principal and parametric constituents in the rule applications of the proof theory and, in this respect, the original strategy of "Kripke" was too restrictive. Moreover, some specific but still unknown properties of $\mathbf{L R}$ that would direct the selection and the order of application of the rules of the proof theory were still wanted. These results on the CM2 were obtained in collaboration with Robert Whaley and John Barlow [RIC 91b].

The second problem may be related to the question of the decidability of (firstorder and modal) logics in two variables that has gained some popularity in computer science lately.

In the case of the logic $\mathbf{R}$, if the undecidability proof relied only on the solution of the word problem for semigroups, we would immediately know that $\mathbf{R}$ in two variables is undecidable and decidable in one variable. But the word problem for semigroups is only one step in A. Urquhart's proof: from the unsolvability of the word problem for semigroups, Hutchinson and Lipshitz have shown that this problem is also unsolvable in modular lattices. These lattices being said appropriate in some way and a countable semigroup being embedded into the multiplicative semigroup of an auxiliary ring of that appropriate lattice, A. Urquhart shows that lattice-like operations are definable on a subset of propositions of $\mathbf{T}, \mathbf{E}$ and $\mathbf{R}$. Moreover, for any proposition of these logics, it is possible to produce a finite set of formulas that guarantees its modularity. Hence the result ${ }^{4}$.

We will return to the word problem for semigroups below. We note that in his complexity proof, A. Urquhart [URQ 90] provides the technique to connect the logics to the algebra i.e. a coding of the language of the logics into that of the algebra, a translation into semi-Thue systems algebra.

\section{Thue systems and semigroups}

In order to see how the logics fit into the algebra, and for later use, let $\Sigma$ be a finite alphabet and $w_{1}, w_{2}, \ldots$ words in $\Sigma^{*}$. A semi-Thue system $S=(\Sigma, R)$ consists in a set of production rules $(R), w_{1} \rightarrow w_{2}$, such that if $w_{1}, w_{2} \in \Sigma^{*}$, then $w_{1} \rightarrow w_{2}(R)$ if there are words $u, v, r, s \in \Sigma^{*}$ s.t. $u=r w_{1} s$ and $v=r w_{2} s$. That is, $v$ is obtained from $u$ by replacement of $w_{1}$ by $w_{2}$. This is called a derivation of $w^{\prime}$ from $w: w_{1} \stackrel{*}{\rightarrow} w_{2}$ means that there is a sequence $w \rightarrow w_{1} \rightarrow w_{i} \rightarrow w_{j} \rightarrow \cdots \rightarrow w_{n} \rightarrow w_{2}$. For example, if $a b \rightarrow a a, b a \rightarrow b b \in R$, then there is a derivation $a b a \rightarrow a b b \rightarrow a a b \rightarrow$ aaa, i.e. $a b a \stackrel{*}{\rightarrow} a a a$.

4. Overviews and analysis of this difficult proof can be found in [AND 92], [PRA 97] and [RES 01]. 
A Thue system $T$, also called a semigroup presentation, is a symmetric semi-Thue system. That is, if $w_{1} \rightarrow w_{2}(R)$, then $w_{2} \rightarrow w_{1}(R)$, that is $w_{1} \leftrightarrow w_{2}(R)$. T generates a congruence, that is, the reflexive transitive closure of $\leftrightarrow$ when, for $x u y \leftrightarrow$ $x v y, u \equiv v(R)$, (i.e. modulo the rules $R$ ), if $u \stackrel{*}{\leftrightarrow} v$. The congruence class of $v$ is $[v]=\left\{u \in \Sigma^{*} \mid u \stackrel{*}{\leftrightarrow} v\right\}$. And the congruence classes of $T$ form a monoid under multiplication and identity, i.e. the monoid presented by $T$.

A. Urquhart's proof [URQ 90] starts with $\mathbf{R}_{\rightarrow \wedge \text { }}$, the implication-conjunctionfusion fragment of $\mathbf{R}$ with propositional constant $t$. A model structure is a triple $M=(0, K, R)$, where $K$ is a set of possible worlds, $0 \in K$ and $R$ is a ternary accessibility relation satisfying the usual postulates of the Routley-Meyer semantics. A valuation in $M$ is a function $V$ assigning a value $V(P) \subseteq K$ to each propositional variables $P$, s.t. if $a \in V(P)$ and $R 0 a b$, then $b \in V(P)$. A formula is valid if $0 \models A$, i.e. $A$ is true at world 0 if it is valid in all models $\mathcal{M}=(M, V)$.

From a commutative-contractive semi-Thue system $S=(\Sigma, R)$, (i.e. such that if $x, y \in \Sigma^{*}$, it contains all productions of form $x y \rightarrow y x$ and $x x \rightarrow x$ respectively), A. Urquhart constructs a model for the logic $\mathbf{R}_{\rightarrow \wedge \circ}$ as follows: define a relational structure $M(S)=\left\langle 0, \Sigma^{*}, R\right\rangle$, where $0 \in \Sigma^{*}$ is the empty string and for $a, b, c \in \Sigma^{*}$, $R a b c$ iff $a b \stackrel{*}{\rightarrow} c(S)$. Then, $M(S)$ is a model structure. Each variable of the logic is correlated to elements of $\Sigma$ : for $a \in \Sigma, P(a)$ is the corresponding propositional variable, and for $s \in \Sigma^{*}, P(s)$ is the corresponding propositional expression. Concatenation in $S$ corresponds to "०", fusion, and the empty string is the propositional constant $t$.

If $a \in \Sigma, b, c, d \in \Sigma^{*}$ in $S$, the canonical model $\mathcal{M}(S)$ associated with $S$ is the model defined on $M(S)$ by $V(P(a))=\left\{s \in \Sigma^{*} \mid a \stackrel{*}{\rightarrow} s(S)\right\}$, and $V(P)=\emptyset$ for uncorrelated variables. Then, $c \models P(b)$ in $\mathcal{M}(S)$ iff $b \stackrel{*}{\rightarrow} c$.

Let $a, b \in \Sigma^{*}$ in $S$, and define a formula $\mathcal{F}(S, a, b)=\left[P\left(c_{1}\right) \rightarrow P\left(d_{1}\right) \wedge \cdots \wedge\right.$ $\left.P\left(c_{n}\right) \rightarrow P\left(d_{n}\right) \wedge t\right] \rightarrow P(b) \rightarrow P(a)$, where $c_{1} \rightarrow d_{1}, \ldots, c_{n} \rightarrow d_{n}$ are the non-contractive non-commutative productions in $S$. Then, $R_{\rightarrow \wedge \circ} \vdash \mathcal{F}(S, a, b)$ iff $a \stackrel{*}{\rightarrow} b(S)$.

Now, relying on Urquhart's reduction of the logics to semi-Thue systems, the decision procedure which, basically, amounts to reducing a proof to some irredundant normal form which is finite by Kripke's lemma or IDP, is similar to the solution of the word problem for commutative semigroups, monoids or Thue-systems. Indeed, these systems are expressible into a semi-Thue system which determines a congruence and equivalence classes. For two classes $[u],[v]$, the system terminates if there is a derivation ending with $[u] \equiv[v]$. By any of the principles equivalent to Kripke or $I D P$, the system terminates. Otherwise there would be an infinite derivation without termination. Since this is exactly what the decision procedure for the logics amounts to, if we could show that the word problem has a constructive proof, we would have a constructive decision procedure for the logics. 


\section{Constructivity}

Although Dickson-Higman-Kripke-Meyer lemmas provide a finiteness or termination condition, their proofs are non-constructive. Nevertheless, the decision procedure and the finiteness condition can be investigated from a constructive point of view through Hilbert's basis theorem in the theory of polynomial rings where Hilbert's theorem has the same finiteness consequence as Dickson's lemma.

Moreover, from a constructive proof of Hilbert's theorem, we obtain a constructive solution of the word problem for (commutative) semigroups and monoids. Given Urquhart's translation of the logic $\mathbf{R}_{\rightarrow \wedge}$ into Thue-systems and semigroups, the decision procedure can be investigated constructively.

We first need to recall the definitions of some basic notions of Algebra that will be used or mentioned in what follows.

A set with an associative and commutative addition and an associative and distributive multiplication is a ring. I.e., a ring $R$ is an additive commutative group with an associative and distributive multiplication operation.

Let $S$ be a set $\left\{x_{1}, \ldots, x_{n}\right\}$ of elements and $R$ a ring. Then, the expression $f(x)=$ $a_{0} x^{0}+a_{1} x^{1}+\cdots+a_{n} x^{n}$ is a polynomial (the sum of its monomial elements) in $x$ indeterminates with coefficients $a_{i} \in R, i=0, \ldots, n$ and $x a=a x$. The highest exponent of $x$ in a polynomial is its degree.

The integers, the rationals, the reals, the integers modulo $n$, the set of all polynomials in $x$ with coefficients in $R$, written $R\left[X_{1}, \ldots, X_{n}\right]$ or simply $R[X]$, are examples of rings.

For all $a \in R, a \neq 0$, and for all $b \in R$, if the equation $a x=b$ has a solution or, equivalently, if the $a$ 's form a group under multiplication, $R$ is called a division ring. Moreover, if $R$ is commutative, it is a field. With respect to the IDP, an interesting example of field is the ring of integers $\bmod n$, when $n$ is a prime number.

If $a b=0$, where $a, b \in R$ (commutative), implies that $a=0$ or $b=0$, i.e., if $R$ has no divisor of zero, $R$ is an integral domain.

If a set $I$ of elements (an additive subgroup) of $R$ is such that if $a, b \in I$ then $a-b \in I$ and for all $a \in I$, for all $r \in R$, ar, $r a \in I$, then $I$ is an ideal.

Then, $R$ has the basis property if every ideal in $R$ is generated by a finite number of elements of $R$.

The following theorem, corresponding to Hilbert's finite basis theorem, defines the notion, fundamental in commutative algebra, of "being Noetherian".

THEOREM 4. - A ring $R$ is noetherian if the following conditions are equivalent:

(i) every ideal in $R$ is finitely generated,

(ii) any ascending chain of ideals is finite (ACC), 
(iii) every ideal in $R$ has a maximal element.

In his work on the complexity of relevant logics, A. Urquhart [URQ 90] relies partly on E. Mayr and A. Meyer [MAY 82], adding the appropriate modifications taking care of the logics. The argument of these authors uses Grete Hermann's results on the complexity of solving system of linear diophantine equations, i.e. polynomials in $K[X]$, where $K$ is some computable field. G. Hermann, a PhD student of E. Noether, explicitly studied Hilbert's theorem in a constructive perspective. It is interesting to note that the title of Hermann's paper [HER 26] translates into "the question of finitely many steps in the theory of polynomial ideals". She partly succeeded, and any later development has relied on her original work.

Essentially, Hermann's results show that if $I$ and $J$ are two finitely generated ideals, one can find a finite set of generators for $I \cap J$ or $I: J$ (the quotient of $I$ by $J$ ), or decide if some member of the ring is also a member of a finitely generated ideal. Moreover she provides some bounds on the complexity of solving systems of polynomial equations.

In the 70's, Seidenberg provided a complete constructive proof of the following version of Hilbert's Theorem:

THEOREM 5. - If $R$ satisfies the ACC and its finitely generated ideals are finitely related, then so does $R[X]$.

First, we need one additional definition: If $R$ is a ring, $M$ an additive abelian group and if there exists a mapping $\phi: R \times M \longrightarrow M$ s.t. if $a \in R$ and $x \in M$, $\phi(a, x)=a x$ and the usual axioms of distributivity and associativity are satisfied, then $M$ is a module. If $R$ is a division ring, then $M$ is a vector space over $R$.

For example, if $R=\mathbb{Z}$, an abelian group is a $\mathbb{Z}$-module, and, remembering the vector spaces underlying the familiar De Morgan monoids in the algebra of Relevant Logics, with $K$, a field, a $K$-vector space is a $R$-module.

Seidenberg [SEI 72] shows that if a ring $R$ satisfies the ACC, then $(i)$ one can find an integer $i$ s.t. $M_{i}=M_{i+1}$ in the chain $M_{1} \subset M_{2} \subset \cdots$ of finitely generated submodules (subgroups of the module closed under multiplication by the $r_{i} \in R$ ) of a finitely based $R$-module $N$ and (ii) $R[X]$ satisfies the ACC.

Then, if $I=\left(f_{1}, \ldots f_{s}\right)$ is a finitely generated ideal in $R[X], n$ a bound on the degree $d$ of the polynomials $f_{i}$ and $\left(g_{1}, \ldots g_{s}\right) \in R[X]$, one can construct a finitely generated submodule $M$ of the $R[X]$-module formed by the $f_{1} g_{1}+\cdots+f_{s} g_{s}$ s.t. $M$ generates $I$. Consequently, $I \cap R[X]=M$ and for any $m$, one can construct $I \cap R\left[X_{1}, \ldots, X_{m}\right]$ and, in particular, $I \cap R$, in a number of steps depending on $n, s$, and $d$. 


\section{Constructive proof of the MP and WP}

In what follows, MP abbreviates the membership problem for polynomial ideals and WP, the word problem for semigroups or Thue systems.

A classical technique of McKinsey [MCK 43] to solve the decision problem for some classes of sentences of first-order logic can be used to solve the word problem for polynomial rings.

Let $L$ be the first-order language associated to a ring. The set of universal sentences (i.e. sentences in prenex normal form without existential quantifiers) which hold in some decidable subsets of the class of rings is recursive.

A conditional sentence has the form $\left(\forall x_{1}, \ldots, \forall x_{n}\right)\left[f_{1}=0 \wedge \cdots \wedge f_{r}=0 \rightarrow\right.$ $f=0$ ], where the $f_{i}$ are terms in the variables $x_{i}$, and each term of the sentence is seen as a polynomial in the variables $x_{i}$. To show that some set of $L$ is recursive, it suffices to show that it is equivalent to some conditional sentence and then to translate the statement that some sentence belongs to that set into a statement concerning the membership of polynomial ideals in the ring of integers, a problem which has a constructive solution.

Indeed, Simmons [SIM 70] shows that, for the ideal $I=\left(f_{1}, \ldots, f_{n}\right)$ in $\mathbb{Z}[X]$ generated by polynomials $f_{i}$, there is an effective procedure to decide the membership of some arbitrary polynomial $f$ in $I$. That is, the membership problem for polynomial ideals is effectively solvable.

In [SIM 80], these results are extended to give a solution to the word problem for Thue systems. This solution translates almost immediately into a solution of the same problem for commutative semigroups and monoids and, moreover, it has a constructive proof.

In their [MAY 82], E. Mayr and A. Meyer give a proof relating the solution of $W P$ in commutative semigroups to the membership problem in polynomial ideals, that is, $W P$ is reducible to $M P$. Hence, given the constructive procedure to solve the membership problem for polynomial rings, there is a constructive procedure to solve the WP for commutative semigroups and monoids.

\subsection{Equivalence of MP and WP}

Let $T=(\Sigma, R)$ be a Thue system with relations $u_{r} \equiv v_{r}(R)$, where the $u_{i}, v_{i} \in$ $\Sigma^{*}$ are words on the alphabet $x_{1}, \ldots, x_{n} \in \Sigma$ as before.

The variables $x_{i}$ can be seen as the indeterminates in a polynomial ring $\mathbb{Q}[X]$ (or $\mathbb{Z}[X]$ ), that is, each word $w \in \Sigma^{*}$ is a monomial in $\mathbb{Q}[X]$.

We can consider polynomials $f_{i}=u_{i}-v_{i}$ of $\mathbb{Q}[X]$ and the ideal $I=\left(f_{1}, \ldots, f_{n}\right)$ in $\mathbb{Q}[X]$. Then, [SIM 80] shows that $u \equiv v$ in $T$ is equivalent to $f \in I$ in $\mathbb{Q}[X]$ (or $\mathbb{Z}[X])$. 
From this, given the reducibility of $W P$ to $M P$ and by Seidenberg construction, there is a constructive procedure to solve the membership problem for polynomial rings. By Simmons result, there is a constructive procedure to solve the WP for commutative semigroups and monoids.

In the non-commutative case, the unsolvability of the word problem for semigroups is a simultaneous result of Post and Markov, published in the JSL in 1946. Now, coming back to undecidable $\mathbf{R}$ and to the second question of section 2 , in order to prove that the word problem for semigroups in 2 generators, or, equivalently, the decision problem for Thue systems on two letters, is recursively unsolvable, Post's result can be used to show that the same holds for any number $n$ of letters. It suffices to show that for each Thue system on the $x_{1}, x_{2}, \ldots, x_{n}$ letters, there is a Thue system on the letters $a, b$ and a solution of the problem for the later system provides a decision for the former.

Indeed, let each of the $x_{i}$ be represented by a string $b a \ldots a b$ with $i$ a's between the $b$ 's and let a Thue system be given by a sequence of corresponding pairs $\left(A_{i}, B_{i}\right)$ of strings and a Thue system on two letters by pairs $\left(A_{i}^{*}, B_{i}^{*}\right)$. If $M$ is a string on $x_{1}, x_{2}, \ldots, x_{n}$ which is mapped into $M^{*}$, a string on $a, b$, then, $M^{*} \equiv N^{*}$ iff $M \equiv N$ in the original system.

The proof given by Marshall Hall, was simplified by Martin Davis who noted that it is already implicitly contained in note 10 of E. Post's paper.

The word problem for semigroups in 2 generators is unsolvable as we have seen, but in one generator, it is solvable given that the one generator case is identical to the commutative case or, as we have also seen, given its equivalence with the $M P$ for polynomial ideals in one indeterminate which is solvable.

\subsection{Back to Dickson et al.}

We can now come back to Dickson's lemma. A consequence of the lemma is that the finitely generated commutative semigroups are finitely presented [RED 65], [CLI 67].

Consider a semigroup presentation, that is, a Thue system $T$, as before, with $u_{i} \equiv$ $v_{i}$, and the ideal $I$ generated by $\left(u_{i} \equiv v_{i}\right)$, where $i \in I$. Then, by Simmons, $u \equiv v$ in $T$ iff $u-v \in I$. If $I$ is finitely generated, there exists some $J \subseteq I$ s.t. $u_{i} \equiv v_{i}$, where the $i \in J$ generate $I$. Hence the presentation is finite.

Starting with Hilbert's theorem, consider an algebra $R M$ constructed from a commutative ring $R$ together with a multiplicative monoid $M$ and let $Q_{i}$ be a congruence in $M$, i.e. the equivalence relations seen as submonoids when $Q_{i} \subseteq M \times M$. Let $I$ be an ideal in $R M$ generated by $(u-v) \in Q$. Then, [EIL 69] shows that $Q_{i}=\left\{(u, v) \mid u, v \in M, u-v \in I\left(Q_{i}\right)\right\}$ if $Q[X]$ and $R M$ are Noetherian. Hence, the ideals as well as the congruences, satisfy the ACC. 
As a corollary, we obtain that any congruence in a finitely generated commutative semigroup is finitely presented [RED 65]. This is equivalent to every finitely generated commutative semigroup is finitely presented (theorem 72 of [RED 65]). By Clifford's proof of Redei's theorem (thm 9.28 in [CLI 67]), who derives the former result from the later, we fall back on Dickson's lemma in its group-theoretic formulation: the set of all minimal elements of a subset $A \subseteq F, F$ a free semigroup, is finite.

Summarizing a few related properties of $\mathbb{N}^{n}$ that, obviously, also hold given the $I D P$, the finitely $k$-generated free commutative monoid $\mathbb{N}_{k}$ is a product of $k$ semigroups of natural numbers. Also, the elements of $\mathbb{N}_{k}$ generated by the first $k$ primes form an integral domain. With unique factorization, it is a free commutative semigroup with $k$ generators. Finally, we have seen that we can also consider the module of linear vector spaces over a computable field. Consider vectors of integers in the monoids $\mathbb{N}^{i}$ seen as subsets of $\mathbb{Q}^{n}$, the vector space of rational numbers over the rationals. They determine systems of homogeneous linear diophantine equations whose sets of positive integral solutions is generated by their sets of minimal elements, their basis, which are finite. And computing in the monoid of positive integers or in a ring amounts to solving systems of linear Diophantines equations, i.e. polynomials.

\section{The missing link: a constructive proof of IDP}

Kripke's lemma or IDP can be proved constructively, but the procedure exposed above does not provide a direct proof.

Proofs of Dickson's lemma are now common place in computer algebra ${ }^{5}$, and several constructive proofs have been proposed, some of them based on J. C. Raoult's principle of open induction [RAO 88] that was advertised and used in T. Coquand [COQ 92] and in his later work.

A property $P$ is inductive if it satisfies the condition $\forall y(\forall x(x>y \Rightarrow P(x)) \Rightarrow$ $P(y))$ and a set, $S$, is Noetherian iff all inductive properties defined on it are satisfied and if the ordering $>$ on $S$ is well-founded, i.e. there are no infinite strictly descending sequences (DCC). Then, if $P \subseteq S, P=S$. It was shown elsewhere that $I D P$ satisfies both conditions.

Following [RAO 88], the closed sets, i.e. those that contain the lub of their directed subsets and are preserved under finite unions and intersection, define a lower topology. Then, a property is open when the set of elements that satisfy the property is open for the lower topology, that is, for all chains $X$, there is a $x$ s.t. $P(\bigvee X) \Rightarrow P(x)$.

An ordering on $S$ is complete if every non-empty >-chain $X$ has a $l u b \bigvee X$ in $S$ and $P \subseteq S$ is open if for all chain $X \subset S$, if $\bigvee X \in P$ then $\exists x \in X$ s.t. $x \in P$. Then,

5. One such proof, mentioned by a referee, that of [BEC 93] as it is used in [LEE 02] where Dickson's lemma is studied in the context of the Mizar project, has been corrected to fit our taste in [RIC 98] where it is proved in the form of the IDP. 
THEOREM 6 (OPEN INDUCTION). - In a complete partially-ordered set (i.e. in which every nonempty chain admits a lub), a property which is inductive and open is true everywhere.

With this principle, IDP can be proven open-inductively if one shows that it defines a complete ordering and that it is open.

First, every non-empty chain $X \in S$ ordered by divisibility has a lub, i.e. $\bigvee X \in$ $S . \mathbb{N}$ ordered by divisibility is a lattice, i.e., for any two elements $x, y \in \mathbb{N}$, there is a $g l b, x \wedge y$ and a $l u b, x \vee y$. Being a chain, either one will do. It suffices to show that every such chain in the set $A_{n}$ of infinite sequences of $\mathbb{N}_{k}$ has a $g l b$. And it is the case from the proof of IDP in [RIC 98].

Second, we have to show that divisibility is an open property. It is the case, indeed, since any subset of the infinite sequence $A_{n}^{\prime}$ has the property and by construction of $\mathbb{N}_{k}$, the integers generated by the first $k$ primes, if any subset has the property, there exists another subset, larger in the ordering that has the property.

Having reached this stage, we see that $I D P$, being already wellfounded, is a too strong property for open induction to be of any interesting use. Nevertheless, if we use an alternative and equivalent interpretation of $I D P$, the situation is different. Indeed, open induction was devised in order to facilitate inductive proofs and proofs by contradiction, in particular, to put under control the orderings of sequences in proofs of Kruskal' theorem, a generalization of Higman's lemma.

In a well-quasi-order, every non-empty subset has only a finite number of minimal elements or, equivalently, for any infinite sequence $s_{n}$ of elements in $S, \exists i, j, i<$ $j$ s.t. $s_{i} \leq s_{j}$. If an infinite sequence satisfies this property, then, a finite initial segment of the sequence already satisfies the property. Thus it suffices to prove that the property is inductive for some quasi-order. We have also seen elsewhere, for example, in [RIC 98], that one of the equivalent formulations of IDP says that If $S$, a set of integer vectors or an infinite sequence of integers, has the IDP, then $S$ is well-quasiordered. Then we can rely on existing constructive proofs of Higman's or Kruskal's theorems.

T. Coquand[COQ 92] has developed interesting methods in the context of formal verification of algorithms, in particular, with respect to Buchberger algorithm seen as a while-loop program. This algorithm terminates by virtue of Dickson or Hilbert's basis theorem and, as it also happens with the size of vectors in $\mathbb{N}_{k}$ in Kripke's lemma or $I D P$, there is no obvious way to control the decrease of the loop. A constructive proof of these principles would then simplify the program verification task. And one way of verifying a program is to give a constructive proof containing an implicit algorithm for the problem to solve, as suggested by Martin-Löf [MAR 82], following Brouwer's ideas.

In order to prove any arbitrary proposition about infinite lists or sequences in a non-well-founded tree called Brouwer's universal spread, Brouwer invented Bar Induction, the principle that says that if some property holds for all the sequences of the 
spread that share some initial segment and if that property is hereditary upwards, then the property bars the spread, i.e. it is satisfied by any sequence of the spread.

\section{Conclusion}

What is gained in using constructive principles, apart from avoiding the use of non-computational reasoning like impredicativity, contradiction, choice... and what is lost, because a constructive proof does not necessarily provide an effective program, are questions worth being considered.

Although they do not provide explicitly a constructive proof of Dickson's lemma there, the authors of [BER 01], mentioned by a referee, work with a refined version of Friedman's $A$-translation (basically, the Gödel procedure of translation of formulas into minimal logic) that allows to translate a classical proof of existence $\exists x B$ into an intuitionnistic proof $\exists^{*} x B$ in order to extract a program. They remark that the classical brute force program doesn't use any information given by the proof, something the extracted program does, having thus "... a chance to be "more efficient"”. Nevertheless, they add that it is not clear yet to see to what that efficiency amounts to as well as to know what efficiency means for programs with infinite sequences as inputs.

In their proof of Higman's lemma based on structural induction and bar induction, the authors of [COQ 93] offer a constructive proof that gives a complete description of the computational content and behavior of the proof. In describing the behavior of the program, some property that tells that the program converges on a bad sequence is open. That means that the program has used only a finite amount of information about that sequence. It has thus an initial segment such that the property holds for any other sequence that has this initial segment. This procedure reminds of Hilbert's $\epsilon$ operator that was supposed to replace or to make sense of some uses of the axiom of choice in mathematics in considering that only finite parts of infinite ideal objects were ever used.

In any case, for our purpose, once we have a proof of the $I D P$, we are interested in its contrapositive form, the corollary: for all $a, b \in A_{n}^{\prime}$, if $a \nmid_{r} b$, then $A_{n}^{\prime}$ is finite. Then, we know that $A_{n}$ will never be larger than the $n$ possible combinations of each $n$-elements vectors compared successively to all the $n^{n}$ remaining possible vectors. And this takes us back to the constructivity proof exposed earlier.

Finally, and this might be another beautiful problem generated by these considerations on Kripke's lemma et alii, there is the first-order theory of the integers generated by the first $k$ primes with relevant divisibility, $\left\langle\mathbb{N}_{k},\left.\right|_{r}\right\rangle$ (or, simply, $\mathbb{N}$ with relevant divisibility [MEY 01]). These theories can be studied from the perspective of model theory. They are still far from being wellknown and sharing the fame of the related Pressburger or Skolem arithmetics, but, from the decidable theory $\langle\mathbb{N}, \leq\rangle$, we already know their decidability and their complexity. In case they ever exhibit any nice and interesting properties and since Meyer's Arithmetics $\left(\mathbf{R}^{\#}\right)$ already exists, we could call them "Meyer's numbers". 


\section{References}

[AND 75] Anderson A. R., Belnap N. D., Eds., Entailment I. The Logic of Relevance and Necessity, Princeton University Press, Princeton, 1975.

[AND 92] Anderson A. R., Belnap N. D., Dunn M., Eds., Entailment II. The Logic of Relevance and Necessity, Princeton University Press, Princeton, 1992.

[BEC 93] Becker T., Weispfenning V., Kredel H., Eds., Gröbner Bases. A Computational Approach to Commutative Algebra, Springer Verlag, New York, 1993.

[Ber 01] Berger U., Schwichtenberg H., Seisenberger M., "The Warshall Algorithm and Dickson's Lemma: Two Realistic Examples of program extraction”, Journal of Automated Reasoning, vol. 26, 2, 2001, p. 205-221.

[CLI 67] Clifford A. H., Preston G. B., The Algebraic Theory of Semigroups, vol. II, American Mathematical Society, Providence, 1967.

[COQ 92] Coquant T., "Constructive Topology and Combinatorics", Myers J. P., O’Donnell M. J., Eds., Constructivity in Computer Science, LNCS 613, Berlin, 1992, Springer, p. 159-164.

[COQ 93] Coquant T., Friedlender D., Heberlin H., “A Proof of Higman's Lemma by Structural Induction,", Unpublished manuscript, 1993, Chalmers University.

[EIL 69] Eilenberg S., Schutzenberger M., "Rational Sets in Commutative Monoids", Journal of Algebra, vol. 13, 1969, p. 173-191.

[HER 26] Hermann G., "Die Frage der endlich vielen Schritte in der Theorie der Polynomideale", Mathematische Annalen, vol. 95, 1926, p. 736-788.

[HIL 90] HiLberT D., "Über die Theorie des algebraischen Formen", Mathematische Annalen, vol. 36, 1890, p. 473-534.

[HIL 93] HILBERT D., "Über die vollen Invariantensystem”, Mathematische Annalen, vol. 42, 1893, p. 313-370.

[LEE 02] LEE G., RUDNICKI P., "Dickson's Lemma”, Journal of Formalized Mathematics, vol. 14, 4, 2002, p. 1-8.

[MAR 82] MARTIN-LÖF P., "Constructive Mathematics and Computer Programming", Logic, Methodology and Philosophy of Science, vol. VI, p. 153-175, North-Holland, 1982.

[MAY 82] MAYR E. W., MeYer A. R., "The Complexity of the Word Problems for Commutative Semigroups and Polynomial Ideals", Advances in Mathematics, vol. 46, 1982, p. $305-329$.

[MCK 43] MCKinsey J., "The Decision Problem for Some Classes of Sentences Without Quantifiers", Journal of Symbolic Logic, vol. 8, 1943, p. 61-76.

[MEY 01] MEYER R. K., "Improved Decision Procedures for Pure Relevant Logics", ANDERSON C. A., et al., Eds., Logic, Meaning and Computation, p. 191-217, Kluwer, Dordrecht, 2001.

[PRA 97] PRAGATI J., “Undecidability of Relevant Logics”, Tr-arp 06-97, 1997, RSISE, Australian National University.

[RAO 88] RAOUlt J. C., "Proving Open Properties by Induction", Information Processing Letters, vol. 29, 1988, p. 19-23.

[RED 65] REDEI L., The Theory of Finitely Generated Commutative Semi-Groups, Pergamon Press, Oxford, 1965. 
[RES 01] Restall G., "Relevant and Substructural Logics", Gabbay D., Woods J., Eds., Handbook of the History and Philosophy of Logic, OUP, Oxford, 2001.

[RIC 91a] RICHE J., "Decidability, Complexity and Automated Theorem Proving in Relevant Logics", PhD thesis, ARP, RSSS, Australian National University, 1991.

[RIC 91b] Riche J., Whaley R., Barlow J., "Massively Parallel Automated Theorem Proving", Workshop on Parallel Processing in AI, IJCAI'91, 1991, p. 178-183.

[RIC 98] Riche J., Meyer R. K., "Kripke, Belnap, Urquhart and Relevant Decidability \& Complexity”, GotTlob G., et al., Eds., Computer Science Logic, CSL’98, LNCS 1584, p. 224-240, Springer, Berlin, 1998.

[SEI 72] Seidenberg A., "Constructive Proof of Hilbert's Theorem on Ascending Chains", Transactions of the American Mathematical Society, vol. 174, 1972, p. 305-312.

[SIM 70] Simmons H., "The Solution of a Decision Problem for Several Classes of Rings", Pacific Journal of Mathematics, vol. 34, 1970, p. 547-557.

[SIM 80] Simmons H., "The Word and Torsion Problems for Commutative Thue Systems", Adian S., Boone W., Higman G., Eds., Word Problems II, Amsterdam, 1980, North Holland, p. 395-400.

[SYL 82] Sylvester J. J., "On Subinvariants, that is, Semi-Invariants to Binary Quantics of an Unlimited Form", American Journal of Mathematics, vol. V, 1882, p. 79-136.

[THI 88] Thistlewaite P., McRobbie M., Meyer R., Automated Theorem-Proving in Non-Classical Logics, John Wiley, New York, 1988.

[URQ 84] URQUhART A., "The Undecidability of Entailment and Relevant Implication", Journal of Symbolic Logic, vol. 40, 1984, p. 1059-1073.

[URQ 90] URquhart A., "The Complexity of Decision Procedures in Relevance Logic", DunN J., Gupta A., Eds., Truth or Consequences, Essays in Honor of Nuel Belnap, p. 6176, Kluwer, Dordrecht, 1990. 